\title{
Resistance to caspase-8 and -9 fragments in a malignant pleural mesothelioma cell line with acquired cisplatin-resistance
}

\author{
V Janson $^{\star, 1,2}$, A Johansson $^{3}$ and K Grankvist ${ }^{1}$
}

Apoptotic cysteine-aspartate proteases (caspases) are essential for the progression and execution of apoptosis, and detection of caspase fragmentation or activity is often used as markers of apoptosis. Cisplatin (cis-diamminedichloroplatinum (II)) is a chemotherapeutic drug that is clinically used for the treatment of solid tumours. We compared a cisplatin-resistant pleural malignant mesothelioma cell line (P31res1.2) with its parental cell line (P31) regarding the consequences of in vitro acquired cisplatin-resistance on basal and cisplatin-induced (equitoxic and equiapoptotic cisplatin concentrations) caspase-3, -8 and -9 fragmentation and proteolytic activity. Acquisition of cisplatin-resistance resulted in basal fragmentation of caspase-8 and -9 without a concomitant increase in proteolytic activity, and there was an increased basal caspase-3/7 activity. Similarly, cisplatinresistant non-small-cell lung cancer cells, H1299res, had increased caspase-3 and -9 content compared with the parental H1299 cells. In P31 cells, cisplatin exposure resulted in caspase-9-mediated caspase-3/7 activation, but in P31res1.2 cells the cisplatininduced caspase-3/7 activation occurred before caspase-8 or -9 activation. We therefore concluded that in vitro acquisition of cisplatin-resistance rendered P31res1.2 cells resistant to caspase-8 and caspase-9 fragments and that cisplatin-induced, initiator-caspase independent caspase-3/7 activation was necessary to overcome this resistance. Finally, the results demonstrated that detection of cleaved caspase fragments alone might be insufficient as a marker of caspase activity and ensuing apoptosis induction.

Cell Death and Disease (2010) 1, e78; doi:10.1038/cddis.2010.54; published online 23 September 2010

Subject Category: Cancer

Cysteinyl aspartate proteinases (caspases) are a large protein family that includes proteins with important roles in cell death pathways, including apoptosis. ${ }^{1,2}$ There are three major pathways of cell death in which caspases are activated; the receptor-mediated pathway with initiator caspase-8, the mitochondrial pathway with initiator caspase- 9 and the cytotoxic lymphocyte-initiated granzyme B pathway. ${ }^{2}$ Granzyme $B$ is a protease that can cleave caspase- 3 and -7 and cleave Bid with subsequent activation of the mitochondrial pathway. ${ }^{2}$ In the other two pathways, appropriate stimuli result in auto-activation of the initiator caspases in large multiprotein complexes with subsequent processing of effector caspases, including caspase-3 and -7, as well as other caspases and effector proteins. ${ }^{1,2}$ All caspases are expressed as inactive precursors, pro-caspases. Activation is usually initiated through proteolytic processing of the pro-caspase and formation of a heterodimer that subsequently is dimerized to form active heterotetramers. ${ }^{1,2}$ Activated caspase-3, -7 and -9 are inhibited by the cytosolic X-linked inhibitor of apoptosis (XIAP), probably the only bona fide caspase inhibitor of the
IAP protein family. ${ }^{3}$ The XIAP domains BIR1 and BIR2 bind to the executor caspases and BIR3 binds to the initiator caspase. $^{3,4}$ The XIAP-caspase interaction is counteracted by proteins released from the mitochondria during activation of the intrinsic pathway, for example, high-temperature requirement protein (HtrA2). ${ }^{5}$ Other IAPs include clAP-1, clAP-2 and survivin, but the role of these proteins in caspase inhibition is not as clear as that of XIAP. ${ }^{3}$ Caspase-8 activity is under the control of FLICE-inhibitory protein isoforms; FLICEinhibitory protein $_{S}$ prevents the cleavage of caspase-8 between the p20 and p10 subunit, FLICE-inhibitory protein inhibits the final cleavage between the prodomain and the p20 subunit of the p43/41 intermediate. ${ }^{6}$

It is recommended that detection of caspase fragments and caspase proteolytic activity is performed simultaneously, to reduce the risk of false negative results. ${ }^{7,8}$ In addition to immunoblotting detection of caspases and their endogenous substrates, for example, PARP cleavage for caspase-3 and -7 activity $^{9}$ or Bid cleavage for caspase- 8 activity, ${ }^{10}$ synthetic substrates can be used in colorimetric or fluorogenic assays

\footnotetext{
1Department of Medical Biosciences, Clinical Chemistry, Umeå University, Umeå, Sweden; ${ }^{2}$ Department of Pathology and Wildlife Diseases, National Veterinary Institute, Uppsala, Sweden and ${ }^{3}$ Department of Odontology, Periodontology, Umeå University, Umeå, Sweden

*Corresponding author: V Janson, Department of Pathology and Wildlife Diseases, National Veterinary Institute, Uppsala SE-751 89, Sweden. Tel: + 46018674282 ; Fax: + 4601867 4363; E-mail: veronica.janson@ sva.se

Keywords: caspase fragmentation; caspase activity; cisplatin-resistance; malignant pleural mesothelioma

Abbreviations: AFC, 7-amino-4-trifluoromethyl coumarin; Bid, Bcl-2 interacting domain death agonist; Bidt, truncated BidBIR1-3, Baculoviral inhibitory repeat 1-3; caspase, cysteinyl aspartate proteinases; c-IAP1-2, cellular inhibitor of apoptosis 1-2; DFF40/CAD, DNA fragmentation factor/caspase-activated DNAse; DFF45/ICAD, DNA fragmentation factor/inhibitor of caspase-activated DNAse; ERK1-2, extracellular-signal regulated kinase; FLICE, old name for caspase-8; HtrA2, high-temperature requirement A2; IAP, inhibitor of apoptosis; NKCC1, the secretory $\mathrm{Na}(+) \mathrm{K}(+) 2 \mathrm{Cl}(-)$-cotransporter; PARP, poly(ADP-ribose) polymerase; XIAP, X-linked inhibitor of apoptosis
}

Received 22.4.10; revised 09.7.10; accepted 02.8.10; Edited by P Salomoni 
for detection of caspase activity. ${ }^{11}$ Caspase-3 activity is often used as a definite marker for apoptosis; however, there is as yet no clear, accepted point-of-no-return for cell death signalling pathways. ${ }^{11}$ It is, therefore, important to determine that caspase activation is followed by the demise of the cells, for example, by determining that the cells have lost plasma membrane integrity or undergone complete fragmentation into discrete bodies. ${ }^{11}$ The fluorescein diacetate cytotoxicity test is a method that determines plasma membrane integrity and cellular viability. ${ }^{12}$ The fluorescein diacetate cytotoxicity test performed in parallel with the detection of oligonucleosomal DNA fragmentation using, for example, terminal deoxynucleotidyl transferase-mediated dUT nick-end labelling assay can be used as an end-point assay for cellular apoptosis. $^{13}$

Cisplatin (cis-diamminedichloroplatinum (II)) is a chemotherapeutic drug used for the treatment of solid tumours, such as malignant mesothelioma and ovarian carcinoma. ${ }^{14}$ The use of cisplatin is limited by severe toxic side-effects, such as nephrotoxicity and neurotoxicity, as well as by intrinsic or acquired tumour resistance. ${ }^{15}$ It is currently considered that the main mechanisms of cisplatin resistance are decreased accumulation of cisplatin in resistant cells; increased intracellular trapping of cisplatin and increased repair of DNA damage or increased tolerance of DNA damage. ${ }^{16}$ Despite this, a plethora of additional resistance mechanisms have been described, including changes in the apoptosis signalling pathways, such as decreased caspase-3 activation ${ }^{17-19}$ and failed activation of caspase-9. ${ }^{18,20}$ To what extent these changes are associated with any of the main mechanisms of cisplatin-resistance mentioned above is not known. However, in a cell model of acquired cisplatin resistance in malignant pleural mesothelioma cells we have previously shown that P31res1.2 cells have reduced NKCC1 activity and disrupted $\mathrm{K}^{+}$-regulation. ${ }^{13}$ It cannot be excluded that these changes may affect caspase activity, as well as, for example, accumulation and intra-cellular trapping of cisplatin. Furthermore, both caspase-3 and caspase-7 can cleave DNA fragmentation factor 45/inhibitor of caspase-activated DNase (DFF45/ICAD) and release active DNA fragmentation factor 40/caspase-activated DNase nuclease (DFF40/CAD), which forms homo-oligomers and generates double-strand breaks in DNA. ${ }^{21}$ Thus, changes in caspase- 3 and -7 activation, and the cellular response to this activation, can be of significance when investigating cisplatin resistance mechanisms. Previously, we have shown that acquisition of cisplatinresistance resulted in a higher basal caspase-3/7 activity, an earlier cisplatin-induced increase in caspase-3/7 activity, but no significant changes in basal levels of nuclear DNA fragmentation. ${ }^{13}$ We have, therefore, determined the effect of acquired cisplatin-resistance on caspase-3, -8 and -9 fragmentation and proteolytic activity in a human malignant mesothelioma cell line of pleural origin, P31 and its cisplatinresistant sub-line P31res1.2. ${ }^{13,22}$ The P31res1.2 cells are about four times more resistant to cisplatin than the P31 cells, with the equitoxic and equiapoptotic cisplatin concentrations being $40 \mathrm{mg} / \mathrm{l}$ and $10 \mathrm{mg} / \mathrm{l}$, respectively. ${ }^{13}$ For comparison we also investigated caspase expression and fragmentation under control conditions in a cisplatin-resistant non-small-cell lung cancer cell line H1299res and its parental cell line,
H1299. These cell lines have a similar difference in cisplatin sensitivity as the P31 and P31res 1.2 cells. ${ }^{23}$ By using timeresolved experiments, we show that this small change in cisplatin-resistance resulted in increased fragmentation of caspase- 8 and -9 , without a concomitant increase in activity. The increased fragmentation appeared to be a consequence of an increased caspase-3/7 activity observed in the P31res 1.2 cells.

\section{Results}

Acquisition of cisplatin-resistance increased basal caspase-3/7 proteolytic activity but not caspase-3 fragmentation. We have previously shown that P31res1.2 cells are four-fold more resistant to cisplatin than their parental P31 cells, with the equitoxic and equiapoptotic cisplatin concentrations being $40 \mathrm{mg} / \mathrm{l}$ and $10 \mathrm{mg} / \mathrm{l}$, respectively. ${ }^{13}$ In this study, we analyzed the cell lines in a time-resolved manner by preparing cell lysates after $30 \mathrm{~min}$, $2 \mathrm{~h}, 6 \mathrm{~h}$ and $24 \mathrm{~h}$ of cisplatin exposure (Figure 1). A caspase$3 / 7$ proteolytic activity assay using the fluorescent substrate DEVD-AFC (Figure 1a) confirmed our previous finding of increased basal caspase-3/7 activity in P31res 1.2 cells. ${ }^{13}$ This was further verified by the increase in PARP fragments under control conditions in the P31 res 1.2 cells (Figure 1b). In a Western blot screening of DFF45/DFF35 content in P31 and P31res1.2 cells, no changes were identified under control conditions (data not shown). In addition, it was not possible to detect any caspase-3 fragments under control conditions in either cell line using western blotting (Figure 1c), despite the use of antibodies from two different companies (not shown). These antibodies could detect caspase-3 fragments, as shown by the positive bands detected after $24 \mathrm{~h}$ of cisplatin exposure in both cell lines (Figure 1c). By using a proteome profiler array, both procaspase-3 protein and cleaved caspase-3 protein was detected under control conditions in P31 and P31res1.2 cells (Figure 1d). The P31res1.2 cells had an increased content pro-caspase-3 protein and, in contrast to the activity assay results, a decreased content of cleaved caspase-3 protein. We therefore used western blot to screen the P31 and P31res1.2 cells for caspase-7 fragmentation, as caspase-7 may substitute for caspase-3. However, both the full-length caspase-7 and the cleaved fragments of caspase-7 were reduced in P31res1.2 cells (data not shown). A 6-h exposure to cisplatin was sufficient to increase the caspase-3/7 proteolytic activity in P31res1.2 cells (Figure 1a), but no change was seen in P31 cells. After $24 \mathrm{~h}$ of cisplatin exposure, both caspase-3/7 proteolytic activity and PARP fragmentation were similar in both cell lines. We therefore concluded that acquisition of cisplatinresistance increased the basal effector caspase activity, but did not change the final response of the effector caspases-3 and -7 to cisplatin exposure. The P31res1.2 cells appeared to be resistant to this increased basal activation, but not to the cisplatin-induced activation, of caspase- $3 / 7$.

To determine whether the basal changes in effector caspase contents were consistent findings after in vitro acquisition of cisplatin resistance, we used western blotting 
a

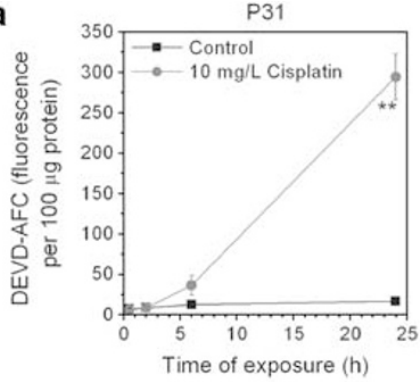

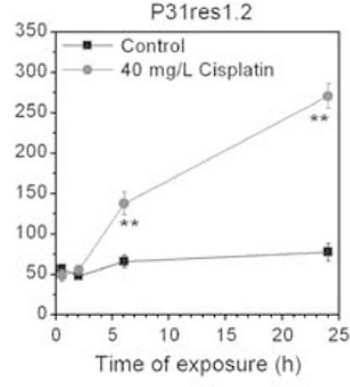

b

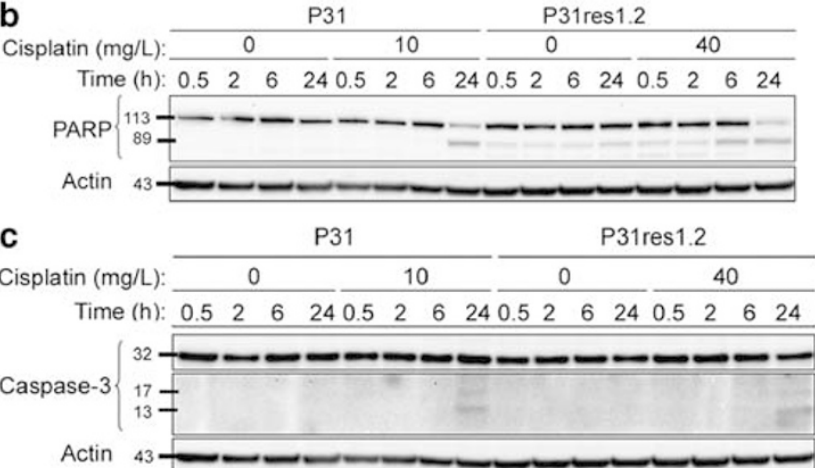

d
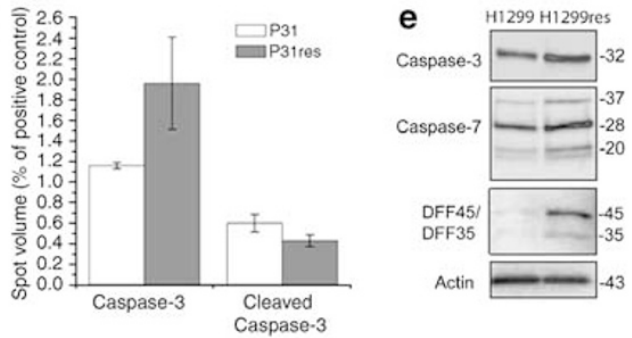

Figure 1 Effector caspase-3/7 activity and caspase-3 fragmentation in malignant pleural mesothelioma cells (P31) and a sub-line with in vitro acquired cisplatin-resistance (P31res1.2) determined in absence and presence of equitoxic and equiapoptotic concentrations of cisplatin, after exposure times as indicated. (a) Caspase-3/7 activity determined in a fluorometric assay using a fluorescenttagged substrate, DEVD-AFC. ${ }^{\star *} P<0.01$, compared with control. Data presented as means \pm S.E.M. of three separate experiments. (b) Western blot of full-length and cleaved PARP as an endogenous indicator of caspase-3/7 activity. The membranes were stripped and reprobed for actin as a protein loading control. Image representative of at least three separate experiments. (c) Activation of caspase-3 determined by western blotting to detect full-length and cleaved versions of caspase3. The membranes were stripped and reprobed for actin as a protein loading control. Image representative of at least three separate experiments. (d) P31 and P31res1.2 content of full-length caspase-3 and cleaved caspase-3 determined with a Proteome profiler array for human apoptosis-related proteins. Data presented as mean$S \pm$ S.E.M. of duplicate analysis. (e) Detection of caspase-3 and caspase-7 by western blotting under control conditions in non-small cell lung cancer cells (H1299) and a sub-line with in vitro acquired cisplatin-resistance (H1299res)

to screen the H1299 and H1299res cells for caspase-3, caspase-7 and DFF45/DFF35 contents under control conditions (Figure 1e). As in the P31res1.2 cells, full-length caspase-3 protein was increased in H1299res cells, but it was again not possible to detect any caspase-3 fragments by western blotting. In contrast to the P31res 1.2 cells (data not shown), the H1299res cells had increased content of DFF45/ DFF35, as well as increased caspase-7 and caspase-7 fragmentation (Figure 1e). Thus, similar to the P31res1.2 cells, the H1299res cells appear to have increased basal expression of effector caspase-3 subsequent to the in vitro acquired cisplatin-resistance. However, the H1299res cells also had increased basal expression and fragmentation of caspase- 7 that was not found in the P31res 1.2 cells.

Acquisition of cisplatin-resistance increased caspase-8 and -9 fragmentation but not proteolytic activity. The fragmentation and proteolytic activity of caspase-8 and -9 was examined in the same manner as for caspase-3. For measuring proteolytic activity, the fluorescent substrate IETD-AFC was used as caspase-8 substrate, and LEHDAFC was used as caspase- 9 substrate. Both caspase- 8 and -9 fragmentations were increased in the P31res 1.2 cells under control conditions (Figure 2a). In particular, the 43 kDa-fragment of caspase- 8 and the 35 and $37 \mathrm{kDa}-$ fragments of caspase- 9 were increased. Despite the increased basal fragmentation of caspase- 8 and -9 there was no increase of basal caspase- 8 or -9 proteolytic activities in P31res1.2 cells compared with P31 cells (Figure 2b). Caspase-8 activity was also investigated by western blotting of Bid and its truncated form tBid, ${ }^{10}$ but there was never any tBid detected in any of the samples (data not shown). A 6-h cisplatin exposure increased only caspase- 9 fragmentation in the P31res 1.2 cells (Figure 2a), but had no effect on the proteolytic activity (Figure $2 \mathrm{~b}$ ). It was only after $24 \mathrm{~h}$ of cisplatin-exposure that caspase- 8 and -9 proteolytic activities were significantly increased in both cell lines. At this timepoint, the amount of caspase- 8 and -9 fragments was similar in both cell lines, consistent with the similar levels of proteolytic activity. It is notable that the p18 fragment of caspase- 8 was barely detectable, and the caspase- 8 activity was also lowest of the activities investigated. This suggested to us that the major initiator caspase in both P31 and P31res 1.2 cells was caspase- 9 , and that the P31res 1.2 cell line had an increased inhibition of, or was resistant to, fragmented caspase- 9 .

To determine whether the basal changes in activator caspase contents were consistent findings after in vitro acquisition of cisplatin resistance, we used western blotting to screen the H1299 and H1299res cells for caspase-8 (not shown) and caspase-9 contents (Figure 2c) under control conditions. In contrast to the P31 and P31res1.2 cells (Figure 2a), no fragments of caspase-8 were identified in the H1299 or H1299res cells (not shown). However, as in the P31res 1.2 cells (Figure 2a), the H1299res cells had increased caspase- 9 content and increased fragmentation of caspase-9, compared with the parental H1299 cells (Figure 2c). Thus, the effect of in vitro acquired cisplatin-resistance on activator caspase- 9 contents and fragmentation appear to be consistent in different cell lines.

Acquisition of cisplatin-resistance increased cIAP-1, cIAP-2, survivin and XIAP, as well as XIAP fragmentation. We investigated the expression levels of four inhibitor-of-apoptosis proteins, clAP-1, clAP-2, survivin and XIAP in a Proteome profiler array. The expression levels of cIAP-2, survivin and XIAP were increased in P31res1.2 cells (Figure $3 \mathrm{a}$ ). We further investigated the possible role of XIAP inhibition of caspase- 9 activity by determining the 

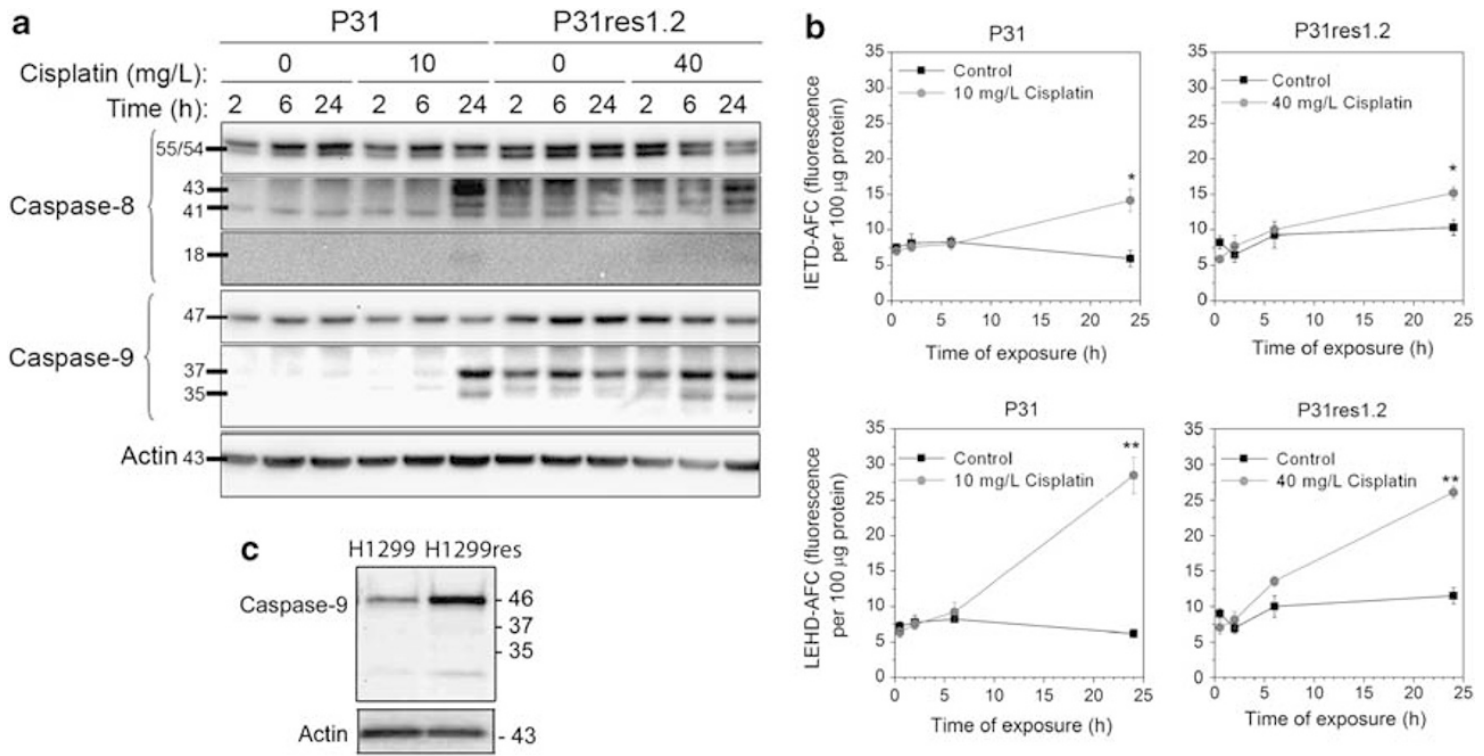

Figure 2 Initiator caspase-8 and -9 fragmentation and activity in malignant pleural mesothelioma cells (P31) and a sub-line with in vitro acquired cisplatin-resistance (P31res1.2), determined in absence and presence of equitoxic and equiapoptotic concentrations of cisplatin, after exposure times as indicated. (a) Western blots of caspase-8 and -9 full-length proteins and cleaved fragments. The membranes were stripped and reprobed for actin as a protein loading control. Image representative of at least three separate experiments. (b) Caspase-8 and -9 activities determined in a fluorometric assay using fluorescent-tagged substrates, IETD-AFC for caspase-8 and LEHD-AFC for caspase-9. Basal activity was determined in control cells maintained without cisplatin and cisplatin-induced activity was determined in cells exposed to equitoxic concentrations of cisplatin as indicated. ${ }^{*} P<0.05,{ }^{* *} P<0.01$, compared to control. Data presented as means \pm S.E.M. of three separate experiments. (c) Detection of caspase-9 by western blotting under control conditions in non-small cell lung cancer cells (H1299) and a sub-line with in vitro acquired cisplatin-resistance (H1299res)

expression and fragmentation of XIAP and through western blotting. Cell lysates were prepared after 2, 6 and $24 \mathrm{~h}$ of cisplatin exposure (Figure $3 b$ ). Both cell lines expressed similar contents of full-length XIAP under control conditions, whereas there was increased XIAP fragmentation with fragments of about $40-45 \mathrm{kDa}$ in the P31res 1.2 cells. There was fragmentation of XIAP also in the P31 cells, but with much lower levels of protein. HtrA2 not only counteracts the XIAP-caspase interaction ${ }^{5}$ but is also reported to mediate cleavage of the protein yielding fragments of $30-45 \mathrm{kDa}^{24}$ Both western blotting (Figure $3 \mathrm{~b}$ ) and Proteome profiler detection (Figure 3a) of HtrA2 expression in whole-cell lysates suggested an increase in expression in the P31res1.2 cells, but western blotting did not show any effect of cisplatin exposure. In contrast, a 24-h cisplatin exposure reduced both the expression of full-length XIAP and XIAP fragmentation in both cell lines (Figure $3 b$ ).

\section{Discussion}

This study shows that a small increase in acquired cisplatinresistance may have a large impact on apoptotic caspase fragmentation and proteolytic activity. We showed that extensive initiator caspase fragmentation, without a concomitant increase in proteolytic activity, occurred in malignant pleural mesothelioma cells with in vitro acquired cisplatinresistance maintained at control conditions. These cells also had increased caspase-3/7 proteolytic activity, but no evidence of caspase- 3 fragmentation. Furthermore, cisplatin exposure resulted first in activation of caspase-3/7, then caspase-9 and to a much lesser extent caspase-8.
We, therefore, concluded that the in vitro acquisition of cisplatin-resistance rendered the P31res1.2 cells resistant to caspase-8 and caspase- 9 fragments and that cisplatininduced caspase-3/7 activation was necessary to overcome this resistance.

The effects of in vitro acquisition of cisplatin-resistance on basal caspase- 3 and -9 contents and fragmentation were similar in H1299res cells and P31res1.2 cells. The H1299res cells are non-small cell lung cancer $\mathrm{H} 1299$ cells with in vitro acquired cisplatin-resistance established in our laboratory. ${ }^{23}$ In contrast to the P31res1.2 cells, the H1299res cells had increased caspase-7 content and fragmentation, indicating that caspase-7 may be upregulated and substitute for caspase- 3 in these cells. Although additional studies are necessary to fully characterize the $\mathrm{H} 1299$ and H1299res cells regarding caspase activity, the results indicate that in vitro acquisition of cisplatin-resistance results in similar changes in basal caspase- 3 and caspase- 9 expression and fragmentation.

Caspase- 9 activation is dependent on the assembly of a functional apoptosome, ${ }^{1,2}$ which in turn is dependent on decreased intracellular $\mathrm{K}^{+}$concentration. ${ }^{25}$ The P31res1.2 cells have deregulated $\mathrm{K}^{+}$transport because of the abrogated NKCC1 activity. ${ }^{13}$ This, and the cisplatin-induced caspase- 9 fragmentation that was detected before any increase in caspase- 9 activity, suggested that the apoptosome assembly or activation was transiently blocked in the P31res1.2 cells. Similar indications of apoptosome dysfunction have been described during cisplatin exposure in nonsmall cell lung cancer cells. ${ }^{26}$ Another possible mechanism is inhibition of caspase- 9 activity by the binding of XIAP. ${ }^{3,4}$ In the P31res1.2 cells, there was increased fragmentation of XIAP, 
a

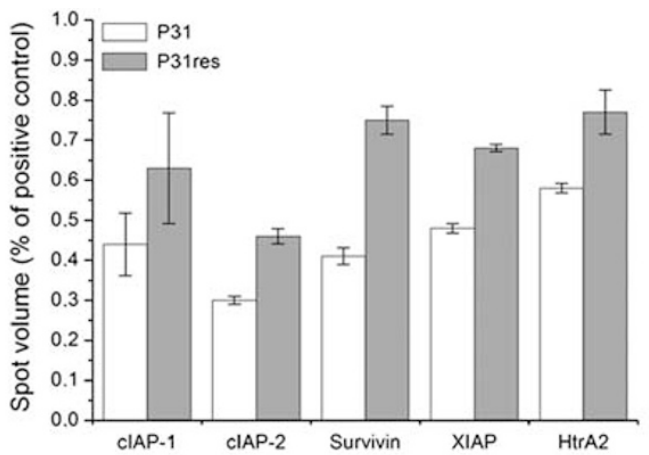

b Cisplatin (mg/L): 0

$\begin{array}{lllllllllllll}\text { Time (h): } & 2 & 6 & 24 & 2 & 6 & 24 & 2 & 6 & 24 & 2 & 6 & 24\end{array}$

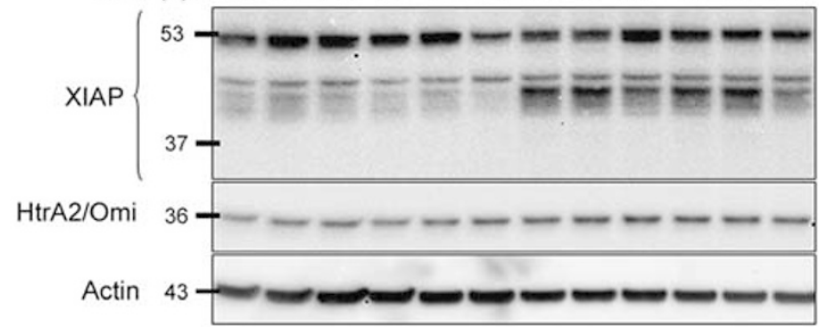

Figure 3 Expression of inhibitor of apoptosis proteins and $\mathrm{HtrA} 2$ in in vitro acquired cisplatin-resistance. (a) P31 and P31res1.2 content of c-IAP1, c-IAP2, survivin, XIAP and HtrA2 determined with a Proteome profiler array for human apoptosis-related proteins. Data presented as means \pm S.E.M. of duplicate analysis. (b) Detection of the caspase-3 and -9 inhibitor XIAP and of the XIAP inhibitor HtrA2XIAP in malignant pleural mesothelioma cells (P31) and a sub-line with in vitro acquired cisplatin-resistance (P31res1.2), determined in absence and presence of equitoxic and equiapoptotic concentrations of cisplatin, after exposure times as indicated. Actin was used as protein loading control. Image representative of three separate experiments

but it is not clear whether these fragments retain the caspase- 9 or caspase-3-inhibiting function of XIAP. A third suggested mechanism for inhibition of caspase- 9 activation is phosphorylation at Thr125 by, for example, extracellular signal-regulated kinase 1 and $2(E R K 1 / 2){ }^{27}$ Although a more detailed investigation is warranted, the involvement of extracellular signal-regulated kinase1/2 is less likely as P31res1.2 cells have about half the amount of phosphorylated extracellular signal-regulated kinase1 and extracellular signal-regulated kinase 2 present in P31 cells. ${ }^{13}$

Caspase- 8 activation occurs in a receptor-associated, death-inducing signalling complex that is under the control of FLICE-inhibitory protein. ${ }^{6}$ In addition, cleaved caspase- 8 is reported to be inhibited by Src-mediated phosphorylation, which inhibits processing of caspase- 8 fragments to generate the $\mathrm{p} 18$ subunit that is the most active subunit. ${ }^{28}$ Although it remains to be verified, our results are consistent with such an inhibition of caspase- 8 activity, as only a very small amount of the p18 subunit was detectable after 24 of cisplatin exposure in both cell lines, concomitant with a slight increase in caspase 8 proteolytic activity.

The increased basal fragmentation of caspase- 8 and -9 in the P31res 1.2 cells was secondary to the increased basal caspase- $3 / 7$ activity. As there was no increased caspase-3 fragmentation in the P31res1.2 cells, it is possible that the in vitro acquisition of cisplatin-resistance had resulted in a chronic increase in caspase-7 translation. ${ }^{29}$ Regardless of the mechanism, our results confirm that there is a threshold of caspase-3/7 activity that must be exceeded for apoptosis to progress, similar to that shown by Rehm et al., $2006 .{ }^{30} \mathrm{~A}$ cisplatin-induced release of active caspase-3/7 from $\mathrm{XIAP}^{1,2,30}$ seemed unlikely as there were no caspase-3/7 fragments detected before $24 \mathrm{~h}$ of cisplatin exposure. However, the decreased XIAP expression after $24 \mathrm{~h}$ of cisplatin expression may have contributed to the increase in caspase-3 and -9 activities, which in turn can stimulate a positive feedback loop with caspase- 3 and -9 cleaving and activating one another. ${ }^{31}$ Caspase-3 and caspase-7 have very similar proteolytic activity, but there are differences in efficiency toward their substrates. ${ }^{32}$ They both have similar efficiency in cleavage of, for example, DEVD-AFC and PARP, whereas caspase- 3 is more efficient in the proteolysis of caspase- 9 and XIAP. ${ }^{32}$ Caspase-3-mediated fragmentation of caspase- 9 occurs at Asp330 and results in a $37-\mathrm{kDa}$ fragment, ${ }^{31}$ the most abundant fragment in the P31res 1.2 cells. In contrast, the XIAP fragments were too large to be the result of caspase3-mediated cleavage. ${ }^{4}$ Thus, another mechanism for the XIAP cleavage seemed more plausible, for example, HtrA2 protease activity, which can result in XIAP fragments of $30-45 \mathrm{kDa},{ }^{24}$ that is, similar to those found in this study. Whether there is a difference in efficiency between caspase-3 and -7 regarding cleavage of caspase- 8 is not known. However, caspase-3-mediated fragmentation of caspase-8 is reported to result in 41 and $43 \mathrm{kDa}$ fragments, ${ }^{33}$ such as those found in this study.

It was notable that three of the four investigated IAP:S, cIAP-2, survivin and XIAP, were increased in the P31res1.2 cells under control conditions. Although the role of CIAP-2 and survivin in caspase inhibition is not as clear as that of XIAP, ${ }^{3}$ it cannot be excluded that they are relevant in the frame of cisplatin-resistance, especially considering the lack of correlation between caspase fragmentation and caspase activity shown in this study. This lack of correlation also emphasizes the importance of combining detection of pro-caspase cleavage and caspase activity when evaluating induced apoptosis by caspase activation. ${ }^{7,8}$ Previously, the main concern has been the risk of false negative results as a consequence of using only caspase fragmentation as marker of activity, based on the observation that, for example,, caspase- 9 can be activated without proteolytic processing. ${ }^{34}$ The results presented here show that analysis of caspase fragmentation also can give false positive results.

To conclude, malignant pleural mesothelioma cells with in vitro acquired cisplatin resistance were resistant to initiator caspase fragmentation with an increased basal caspase-3/7 activity and initiator-caspase independent cisplatin-induced activation of caspase-3/7. The parental cell line, which was less resistant to cisplatin, appeared to have caspase-9mediated, cisplatin-induced caspase-3 activity. Thus, acquisition of cisplatin-resistance may cause resistance to caspase activation and activity, particularly through the caspase 9-mediated pathway. Finally, the lack of correlation between caspase fragmentation and caspase activity showed that detection of fragments alone is insufficient as a marker of caspase activity and therefore apoptosis induction. 


\section{Materials and Methods}

Cell culture. A human malignant mesothelioma cell line of pleural origin (P31 $)^{22}$ and its cisplatin-resistant sub-line (P31res1.2 with a $4 \times$ increased $\left.\mathrm{LD}_{50}\right)^{13}$ was used. Both cell lines were propagated at $37^{\circ} \mathrm{C}$ in a humidified atmosphere containing $5 \% \mathrm{CO}_{2}$, grown as monolayer cultures in Eagle's minimal essential culture medium with Earl's salts supplemented with $10 \%$ fetal calf serum, $200 \mu \mathrm{mol} / \mathrm{l}$ L-glutamine, gentamicine and, in the case of the P31res 1.2 cells, $1.2 \mathrm{mg} / \mathrm{l}$, cisplatin. The cells were exposed to equitoxic and equiapoptotic concentrations of cisplatin, $10 \mathrm{mg} / \mathrm{l}$ for P31 cells and $40 \mathrm{mg} / \mathrm{l}$ for P31res 1.2 cells for $0.5,2,6$ or $24 \mathrm{~h}$ and thereafter analyzed regarding caspase fragmentation and proteolytic activity. ${ }^{13}$

Western blotting. Western blotting was performed as described previously. ${ }^{13}$ In brief, the cells were harvested by trypsination immediately after exposures, lyzed in lysis buffer $(50 \mathrm{mmol} / \mathrm{I}$ Tris $7.5,1 \%$ Triton X-100,150 mmol// NaCl and $1 \mathrm{mmol} / \mathrm{l}$ $\mathrm{NaF}, 1$ complete protease inhibitor EDTA-free cocktail tablet to $50 \mathrm{ml}$ buffer, Roche, Manheim, Germany) and stored at $-80^{\circ} \mathrm{C}$ until analysis. A BCA protein assay reagent kit was used according to the manufacturers' instruction (Pierce Biotechnology, Rockford, IL, USA) to determine the total protein content of lysates. Lysates from two separate experiments were loaded on the same gel. An amount of $20 \mu \mathrm{g}$ proteins per sample were separated with SDS-PAGE and transferred to PVDF-membranes (Bio-Rad Laboratories, Hercules, CA, USA). After incubation with appropriate primary and secondary antibodies, the protein bands were visualized (ECL Advance Western Blotting Detection Kit, GE Healthcare, Little Chalfont, UK) and quantified with the Chemidoc XRS system and Quantity One 1-D Analysis software (Bio-Rad Laboratories). All membranes were stripped ${ }^{17}$ and probed with actin antibody (Millipore, Billerica, MA, USA) to use actin bands as protein loading control. The primary antibodies used were against caspase-3 (Cell Signaling Technology, Boston, MA, USA), caspase-8 (Alexis Biochemicals, Lausen, Switzerland), caspase-7, caspase-9, PARP, DFF45/35, Bid, HtrA2 and XIAP (all Cell Signaling Technology).

Proteome profiler array. A Proteome profiler array for human apoptosisrelated proteins (R\&D Systems, Minneapolis, MN, USA) was used for the parallel determination of relative levels of caspase-3 (full-length and cleaved fragments), $\mathrm{HtrA} 2$ and four inhibitors of apoptosis proteins (IAPs) in P31 and P31res1.2 cells. The array was used according to the manufacturer's instructions. Briefly, cells plated on $15-\mathrm{cm}$ culture plates were grown to about $80 \%$ confluence, washed with cold phosphate-buffered saline and lyzed with the kit-included lysis buffer. A BCA protein assay reagent kit was used according to the manufacturers' instruction (Pierce Biotechnology) to determine the total protein content of lysates. The array membranes were incubated overnight with $300 \mu \mathrm{g}$ protein per membrane and processed according to manufacturer's instructions. The array spots were detected (ECL Advance Western Blotting Detection Kit, GE Healthcare), visualized and quantified with the Chemidoc XRS system and Quantity One 1-D Analysis software (Bio-Rad Laboratories). For quantification the spot volume was determined, corrected for background and expressed as percent of positive control.

Caspase activity fluorometric assay. To determine caspase activity, fluorometric caspase activity assays (R\&D Systems) were used according to the manufacturers' instructions, as described previously. ${ }^{13}$ In brief, the cells were lyzed immediately at the end of exposures (kit-included lysis buffer) and the supernatant was stored at $-80^{\circ} \mathrm{C}$ until analysis. A BCA protein assay reagent kit was used according to the manufacturers' instruction (Pierce Biotechnology) to determine the total protein content of lysates. $100 \mu \mathrm{g}$ proteins were incubated with fluorescent substrate; DEVD-AFC for caspase-3/7, IETD-AFC for caspase-8 and LEHD-AFC for caspase- 9 and fluorescence was determined in a LS55 Luminescence spectrometer (PerkinElmer, Waltham, MA, USA). 400 and $505 \mathrm{~nm}$ was used for excitation and emission, respectively. The results were corrected for background fluorescence, and the data is presented as fluorescence per $100 \mu \mathrm{g}$ total protein.

Chemicals. Culture medium (Eagle's MEM with Earle's salts) and L-glutamine were from Gibco Ltd. Fetal calf serum was purchased from Biochrom KG (Berlin, Germany), cisplatin (CAS 15663-27-1) was from Bristol-Myers Squibb (New York, NY, USA). All other chemicals were of analytical grade.

Statistical analysis. All results are presented as means \pm S.E.M., and for statistical analysis, the SPSS 12.0.1 for Windows program was used. When normal distribution could be assumed, an independent Student's $t$-test was used for effect of exposure time and cell type. When normal distribution could not be assumed, the
Wilcoxon's Signed Ranks test were used for effect of cisplatin concentration, and the Mann-Whitney $U$-test for the effect of exposure time and cell type. $P$-values from two-sided tests were used, and the level of significance for rejecting the null hypothesis of zero effect was taken to be $p \leq 0.05$.

\section{Conflict of interest}

The authors declare no conflict of interest.

Acknowledgements. The authors thank Jasmin Moharer for technical assistance. The study was supported by the Lions foundation, the Swedish Cancer Society, the County Council and the Faculty of Medicine, Umeå University, Umeå, Sweden.

1. Kurokawa M, Kornbluth S. Caspases and kinases in a death grip. Cell 2009; 138 838-854.

2. Taylor RC, Cullen SP, Martin SJ. Apoptosis: controlled demolition at the cellular level. Nat Rev Mol Cell Biol 2008; 9: 231-241.

3. Eckelman BP, Salvesen GS, Scott FL. Human inhibitor of apoptosis proteins: why XIAP is the black sheep of the family. EMBO Rep 2006; 7: 988-994.

4. Deveraux QL, Leo E, Stennicke HR, Welsh K, Salvesen GS, Reed JC. Cleavage of human inhibitor of apoptosis protein XIAP results in fragments with distinct specificities for caspases. EMBO J 1999; 18: 5242-5251.

5. Vaux DL, Silke J. Mammalian mitochondrial IAP binding proteins. Biochem Biophys Res Commun 2003; 304: 499-504.

6. Wajant H. Targeting the FLICE Inhibitory Protein (FLIP) in cancer therapy. Mol Interv2003; 3: $124-127$.

7. Kaufmann SH, Lee S-H, Meng XW, Loegering DA, Kottke TJ, Henzing AJ et al. Apoptosisassociated caspase activation assays. Methods 2008; 44: 262-272.

8. Vaculova A, Zhivotovsky B. Caspases: determination of their activities in apoptotic cells Methods Enzymol 2008; 442: 157-181.

9. Inoue S, Browne G, Melino G, Cohen GM. Ordering of caspases in cells undergoing apoptosis by the intrinsic pathway. Cell Death Differ 2009; 16: 1053-1061.

10. Ho PK, Hawkins CJ. Mammalian initiator apoptotic caspases. FEBS J 2005; 272 5436-5453.

11. Kroemer G, Galluzzi L, Vandenabeele P, Abrams J, Alnemri ES, Baehrecke EH et al. Classification of cell death: recommendations of the Nomenclature Committee on Cell Death 2009. Cell Death Differ 2009; 16: 3-11.

12. Lindhagen $E$, Nygren $P$, Larsson $R$. The fluorometric microculture cytotoxicity assay Nat Protoc 2008; 3: 1364-1369.

13. Janson V, Andersson B, Behnam Motlagh P, Engstrom KG, Henriksson R, Grankvist K Acquisition of cisplatin-resistance in malignant mesothelioma cells abrogates $\mathrm{Na}^{+}, \mathrm{K}^{+}, 2 \mathrm{Cl}$ -cotransport activity and cisplatin-induced early membrane blebbing. Cell Phys Biochem 2008; $21: 45-56$.

14. Kelland $\mathrm{L}$. The resurgence of platinum-based cancer chemotherapy. Nat Rev Cancer 2007; 7: 573-584.

15. Giaccone G. Clinical perspectives on platinum resistance. Drugs 2000; 59 (Suppl 4): 9-17; discussion $37-18$

16. Borst $\mathrm{P}$, Rottenberg $\mathrm{S}$, Jonkers J. How do real tumors become resistant to cisplatin? Cell Cycle 2008; 7: 1353-1359.

17. Ikuta K, Takemura K, Kihara M, Naito S, Lee E, Shimizu E et al. Defects in apoptotic signal transduction in cisplatin-resistant non-small cell lung cancer cells. Oncol Rep 2005; 13 1229-1234.

18. Okouoyo S, Herzer K, Ucur E, Mattern J, Krammer PH, Debatin KM et al. Rescue of death receptor and mitochondrial apoptosis signaling in resistant human NSCLC in vivo. Int J Cancer 2004; 108: 580-587.

19. Yang $X$, Zheng F, Xing H, Gao Q, Wei W, Lu Y et al. Resistance to chemotherapy-induced apoptosis via decreased caspase-3 activity and overexpression of antiapoptotic proteins in ovarian cancer. J Cancer Res Clin Oncol 2004; 130: 423-428.

20. Mueller T, Voigt W, Simon H, Fruehauf A, Bulankin A, Grothey A et al. Failure of activation of caspase-9 induces a higher threshold for apoptosis and cisplatin resistance in testicula cancer. Cancer Res 2003; 63: 513-521.

21. Widlak $P$, Garrard WT. Discovery, regulation, and action of the major apoptotic nucleases DFF40/CAD and endonuclease G. J Cell Biochem 2005; 94: 1078-1087.

22. Marklund SL, Westman NG, Lundgren E, Roos G. Copper- and zinc-containing superoxide dismutase, manganese-containing superoxide dismutase, catalase, and glutathione peroxidase in normal and neoplastic human cell lines and normal human tissues. Cancer Res 1982; 42: 1955-1961.

23. Johansson D, Andersson C, Moharer J, Johansson A, Behnam-Motlagh P. Cisplatininduced expression of $\mathrm{Gb} 3$ enables verotoxin-1 treatment of cisplatin resistance in malignant pleural mesothelioma cells. Br J Cancer 2010; 102: 383-391.

24. Vande Walle L, Lamkanfi M, Vandenabeele $P$. The mitochondrial serine protease HtrA2 Omi: an overview. Cell Death Differ 2008; 15: 453-460. 
25. Karki P, Seong C, Kim JE, Hur K, Shin SY, Lee JS et al. Intracellular K(+) inhibits apoptosis by suppressing the Apaf-1 apoptosome formation and subsequent downstream pathways but not cytochrome c release. Cell Death Differ 2007; 14: 2068-2075.

26. Checinska A, Hoogeland BS, Rodriguez JA, Giaccone G, Kruyt FA. Role of XIAP in inhibiting cisplatin-induced caspase activation in non-small cell lung cancer cells: a small molecule Smac mimic sensitizes for chemotherapy-induced apoptosis by enhancing caspase-3 activation. Exp Cell Res 2007; 313: 1215-1224.

27. Allan LA, Clarke PR. Apoptosis and autophagy: Regulation of caspase- 9 by phosphorylation. FEBS J 2009; 276: 6063-6073.

28. Cursi S, Rufini A, Stagni V, Condo I, Matafora V, Bachi A et al. Src kinase phosphorylates Caspase-8 on Tyr380: a novel mechanism of apoptosis suppression. EMBO J 2006; 25 : 1895-1905

29. Yang C, Kaushal V, Haun RS, Seth R, Shah SV, Kaushal GP. Transcriptional activation of caspase- 6 and -7 genes by cisplatin-induced $p 53$ and its functional significance in cisplatin nephrotoxicity. Cell Death Differ 2008; 15: 530-544.

30. Rehm M, Huber HJ, Dussmann H, Prehn JH. Systems analysis of effector caspase activation and its control by $\mathrm{X}$-linked inhibitor of apoptosis protein. EMBO J 2006; 25 4338-4349.
31. Slee EA, Harte MT, Kluck RM, Wolf BB, Casiano CA, Newmeyer DD et al. Ordering the cytochrome $c$-initiated caspase cascade: hierarchical activation of caspases-2, $-3,-6$, $-7,-8$, and -10 in a caspase-9-dependent manner. J Cell Biol 1999; 144: 281-292.

32. Walsh JG, Cullen SP, Sheridan C, Luthi AU, Gerner C, Martin SJ. Executioner caspase-3 and caspase-7 are functionally distinct proteases. Proc Natl Acad Sci USA 2008; 105: 12815-12819.

33. Guerrero AD, Chen M, Wang J. Delineation of the caspase-9 signaling cascade. Apoptosis 2008; 13: 177-186.

34. Stennicke HR, Deveraux QL, Humke EW, Reed JC, Dixit VM, Salvesen GS. Caspase-9 can be activated without proteolytic processing. J Biol Chem 1999; 274: 8359-8362.

Cell Death and Disease is an open-access journal published by Nature Publishing Group. This work is licensed under the Creative Commons Attribution-Noncommercial-No Derivative Works 3.0 Unported License. To view a copy of this license, visit http://creativecommons.org/licenses/by-nc-nd/3.0/ 